\title{
Lesson from a Cecal Phlegmon: From an Acute Appendicitis to a Solitary Cecal Diverticulitis
}

\author{
Nuno Gonçalves ${ }^{1,2^{*}}$, José Pedro Pinto ${ }^{1}$, Ricardo Pereira ${ }^{1}$, Joaquim Costa Pereira ${ }^{1}$ \\ Corresponding author: \\ Nuno Gonçalves \\ Serviço Cirurgia Geral \\ 'Serviço Cirurgia Geral, Hospital de Braga, Braga, Portugal \\ 2Escola de Medicina, Universidade do Minho, Braga, Portugal
}

Hospital de Braga, Braga, Portugal

Tel.: +351918280593

E-mail: nunogsilvagoncalves@gmail.com

\begin{abstract}
A cecal phlegmon can be a difficult situation for a surgeon, since the treatment strategy must be decided with uncertainty of the phlegmon's origin: benign or malignant. The most common causes are cecal diverticulitis, acute appendicitis and cecal carcinoma. More rarely, it can be caused by a appendicular diverticulitis (1). In this case, we present a case of a cecal diverticulum, which is a rare but benign medical condition, in a 52-year-old man who presented to the emergency department with a 3-day constant abdominal pain in the right lower quadrant with rebound and with an increase of the inflammatory analytical levels. An abdominal ultrasound showed findings compatible with an acute appendicitis and the patient was proposed to a laparoscopic appendectomy. Intraoperatively, a cecal phlegmon was found and an ileocecal resection with primary anastomosis was conducted. Histopathological findings confirmed a rare solitary cecum diverticulitis.
\end{abstract}

Key words: cecal diverticulitis, diverticulosis, acute appendicitis, surgery, cecal phlegmon

\section{INTRODUCTION}

Colonic diverticulosis was first described by Portier in 1912. Its prevalence is difficult to assess since most individuals remain asymptomatic during their life. Diverticulosis is the most frequent structural alteration diagnosed at colonoscopy. In the western world, $85 \%$ of diverticulosis is found on the left/ sigmoid colon, while it is rarely found on the cecum, contrasting with oriental population, where the incidence is opposite. Cecal diverticula can either be congenital or acquired. Congenital cecal diverticula are true diverticula, composed of all layers of the colon, and develop during the $6^{\text {th }}$ week of gestation. Acquired diverticula do not contain all layers and are similar to those from the sigmoid colon (2). From all diverticular formations in the cecum, $80 \%$ are located less than $2.5 \mathrm{~cm}$ from the ileocecal valve and about $50 \%$ are found in the anterior margin $(3,4)$.

Cecum diverticulitis $(C D)$ is a rare cause of acute appendicitis-like clinic, diagnosed in around 1:300 appendectomies worldwide(2,5-7). Since it mimics the medical history and physical examination of acute appendicitis (AA) and can even cause radiological imaging misinterpretation, patients are 
usually proposed to undergo surgical treatment. More than $70 \%$ of the patients diagnosed with CD were previously diagnosed with AA $(5,8)$. In those cases, only intraoperatively, the surgeon can unfold the correct diagnosis. However, sporadically the surgical team is faced with a cecal phlegmon, difficult to assess its true origin, which represents a challenge, in which they must decide whether it is a benign or malignant condition and based on that choose a more conservative approach such as an appendectomy with postoperative antibiotics therapy or choose a more radical approach such as a right hemicolectomy. In this article, we report a case of a clinical and imagological AA, proposed to laparoscopic appendectomy that turned out to be a $C D$ and discuss the different approaches to a cecal phlegmon.

\section{CASE REPORT}

A 52-year-old man presented to the emergency department with a 3-day constant abdominal pain in the right lower quadrant (RLQ), without migration, associated with anorexia. The patient denied fever, nausea, vomits, diarrhea or any bowel habits alteration. The clinical examination revealed pain on palpation of the RLQ, with a positive Blumberg sign. There were no medical relevant priors. Laboratory tests demonstrated a normal leukocytes value with an elevation of C-reactive protein $(41 \mathrm{mg} / \mathrm{dL}$, for a normal range $<5$ $\mathrm{mg} / \mathrm{dL}$ ). An abdominal ultrasound was conducted showing a heterogeneous formation, hypoechogenic, with $34 * 23 \mathrm{~mm}$, associated with small adjacent hypoechogenic areas with correspondence with acute appendicitis, eventually with a perforation.

The patient was proposed to a laparoscopic appendectomy, which he agreed. Intraoperatively, the appendix had an innocent presentation without any inflammatory signs, however, adjacent to the appendix a cecal phlegmon was present (fig. 1). The surgical team considered that the most likely etiology was an inflammatory mass but a malignant cause could not be excluded. For this reason, a laparoscopic release of the right colon was conducted, followed by a median periumbilical minilaparotomy, an ileocecal resection and a mechanical latero-lateral isoperistaltic anastomosis. The patient was discharged two days after with full recovery.

\section{Outcome and follow up}

A six-month follow-up did not found any morbidity. The histopathological report showed a true solitary cecal diverticulum with a contained perforation (fig. 2).
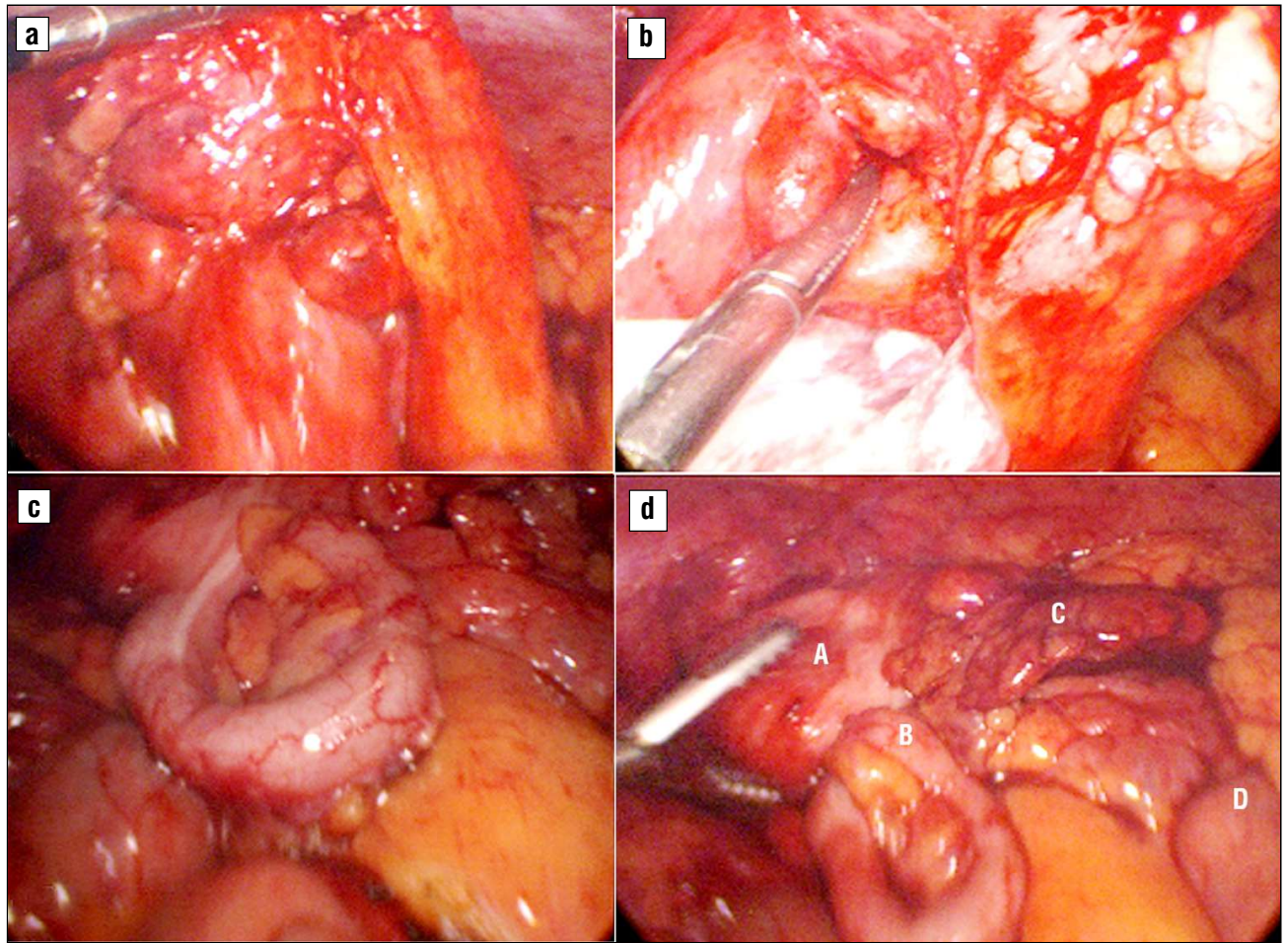

Figure 1 - Intraoperative views. (a) - View of the cecal phlegmon; (b) - View of the cecal perforation;

(c) - View of the morphological normal Vermiform Appendix; (d) - Anatomic view of diferrent structures (A - Cecum, B - Vermiform Appendix, C - Cecal diverticulum; D - Ileum) 


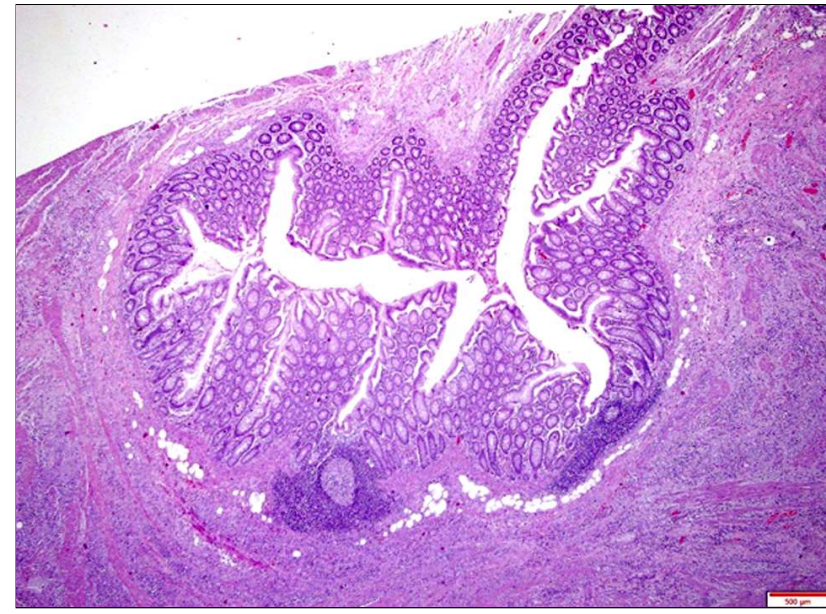

Figure 2 - Histological findings demonstrating a true solitary cecal diverticulum

\section{DISCUSSION}

Colonic diverticulosis is the presence of small bulging pouches that form in the wall of the colon. They can either be true diverticula, when it is formed by all layers of the colon, or false diverticula which do not contain the muscular layers or adventitia, only involving the submucosa and mucosa (much more common than true diverticula).

While the most recent literature states that colonic diverticulosis appears in $8.5 \%$ of the world population $(5,7)$, right colonic diverticula only accounts for $1.5 \%$ of those (9), which is believed to be approximately around $1 / 1,000,000(10)$. The average age of presentation is around 43 years with a $3: 2$ male predominance $(4,11)$. Adding to the low incidence of right colonic diverticula in western countries, the clinical presentation of $C D$ is a right iliac pain in $99 \%$ of patients (9), which usually leads to a misdiagnosed assumption of an AA or other causes of right iliac fossa pathology. Historically, cecal diverticulosis was diagnosed using barium enema, however the interpretation is often inconclusive. Nowadays, colonoscopy remains the gold standard, but should not be used in acute episodes (12).

Diverticulitis is one of the most common emergency department admission reasons. The classical clinical signs are pain in the lower left quadrant in the absence of vomits, with an increase in the white cell blood count and C-reactive protein.

There are also studies attempting to differentiate $C D$ from AA. They report a more prolonged clinic of pain, located only in the right lower quadrant in the absence of other symptoms, while in AA patients the pain begins in the center of the abdomen migrating to the RLQ accompanied with anorexia, nausea or vomits.
Nevertheless, physical examination frequently fails to differentiate these conditions (13).

Imaging plays a crucial important role in the differential diagnosis. It is estimated that ultrasound has a sensitivity of $91.3 \%$ and a sensitivity of $99.8 \%$ with an overall accuracy of $99.5 \%$ in the diagnosis of cecal diverticulitis. Recent literature clearly states that CT scan is superior to ultrasound (4), with a specificity and sensitivity values around 98\% (11). There are some CT features that can help in the differentiation of benign from malignant causes, where there is a focal asymmetric and eccentric bowel wall thickening (with less than $5 \mathrm{~cm}$ ). It can also be detected homogeneous contrast enhancement due to infiltration of a tumor mass, or a heterogeneous area of low attenuation from ischemia and necrosis (14). Magnetic Resonance (MRI) has been demonstrated to be a useful tool on pregnant and young patients (4).

As for macroscopic appearance, two major types of cecal diverticulitis are described: The most common type consists of an inflamed projection of the cecal wall, while the other variant presents as a large indurated phlegmon and is difficult to distinguish from a perforated carcinoma.

It is also reported that right side diverticula have low complications rate (15), present in less than 15\% patients $(2,16)$. The most reported complications are phlegmon, abscess formation, fistula, obstruction, hemorrhage, torsion and perforation (10).

When intraoperatively the surgeon is faced with a phlegmon, efforts must be done to try to differentiate the etiology, either benign or malignant. The most common differential diagnosis of a cecal phlegmon are acute appendicitis, right sided diverticulitis, inflammatory bowel disease (mainly crohn's disease), inflammatory pelvic disease or more rarely ileocecal tuberculosis. There are some reports that suggest performing a colonoscopy during surgery in order to assess presence of diverticula or malignant signs, however the risk of perforation of an already inflamed bowel cannot be underestimated (17).

There are no proper guidelines regarding treatment options when facing this dilemma since there are no sufficient randomized clinical trials. If the diagnosis is made preoperatively in an uncomplicated $C D$, the standard treatment is conservative, using analgesics and anti-inflammatory drugs to relieve pain. If uncomplicated $C D$ is only verified on the operating table, an incidental appendectomy alone or accompanied by a diverticulectomy, depending on the surgeon's expertise and choice, is usually the procedure of choice $(10,13)$. In complicated diverticulitis, surgical treatment 
is the recommendation. The literature states that ileocecal resection with primary anastomosis is the procedure of choice when the suspicion of malignancy is not exceedingly high, opting for right hemicolectomy in those cases. Recent literature reports no morbidity in patients with $C D$ submitted to ileocecal resection, in opposition with right hemicolectomy, associated with an increased surgical time and morbidity rate $(12,13$, 18,19). However, careful intraoperative assessment including examination of the resected specimen is essential to exclude malignancy, which would require a right hemicolectomy.

\section{CONCLUSION}

Cecal diverticulitis is a very uncommon diagnosis of right lower quadrant pain, being more prevalent in asian countries, and it should be part of the differential diagnosis of $A A$, since it mimics its clinic. A high-grade diagnostic suspicion is needed to identify preoperatively possible uncomplicated $C D$ eligible for the conservative approach while for the complicated cases, the surgical option is the gold standard treatment. A cecal phlegmon is a dilemma for the surgeon, in which he must decide on a more conservative or aggressive approach depending on the probability of a malignant etiology. The decision should be based on the clinical presentation, the intraoperative findings and the surgical team experience.

\section{Learning Points/Take-home Messages}

- In case of a right iliac pain, and especially in Asian patients, a differential diagnosis of a cecal diverticulitis should be conducted when assuming an acute appendicitis.

- In case of a preoperative correct diagnosis of cecal diverticulitis, medical treatment should be preferred.

- In complicated cecum diverticulitis, surgery is the gold standard treatment.

\section{Declarations of interest: none}

\section{Ethics Approval}

The case study was done by following the ethical norms of scientific research and the principles anonymity a confidentiality.

\section{REFERENCES}

1. Constantin VD, Carâp A, Nica A, Smaranda A, Socea B. Appendiceal Diverticulitis - A Case Report. Chirurgia (Bucur). 2017;112(1):82-85.

2. Koshy RM, Abusabeib A, Al-Mudares S, Khairat M, Toro A, Di Carlo I. Intraoperative diagnosis of solitary cecal diverticulum not requiring surgery: is appendectomy indicated? World J Emerg Surg. 2016;11:1.

3. Abogunrin FA, Arya N, Somerville JE, Refsum S. Solitary caecal diverticulitis--a rare cause of right iliac fossa pain. Ulster Med J. 2005;74(2):132-3.

4. Mudatsakis N, Nikolaou M, Krithinakis K, Matalliotakis M, Politis N, Andreadakis E. Solitary cecal diverticulitis: an unusual cause of acute right iliac fossa pain-a case report and review of the literature. Case Rep Surg. 2014;2014:131452.

5. Karatepe 0, Gulcicek OB, Adas G, Battal M, Ozdenkaya Y, Kurtulus I, et al. Cecal diverticulitis mimicking acute Appendicitis: a report of 4 cases. World J Emerg Surg. 2008;3:16

6. Griffiths EA, Bergin FG, Henry JA, Mudawi AM. Acute inflammation of a congenital cecal diverticulum mimicking appendicitis. Med Sci Monit. 2003;9(12):CS107-109.

7. Papapolychroniadis C, Kaimakis D, Fotiadis P, Karamanlis E, Stefopoulou M, Kouskouras K, et al. Perforated diverticulum of the caecum. A difficult preoperative diagnosis. Report of 2 cases and review of the literature. Tech Coloproctol. 2004;8(1):s116-8.

8. Lane JS, Sarkar R, Schmit PJ, Chandler CF, Thompson JE. Surgical approach to cecal diverticulitis. J Am Coll Surg. 1999;188(6): 629-34; discussion 634-635.

9. Yuksel A, Civil O, Colakoglu MK, Sumer F, Eruyar AT. Solitary cecal diverticulitis, a rare cause of right lower quadrant pain: Four cases. North Clin Istanb. 2018;5(2):148-52.

10. Ezer A, Parlakgumus A. Solitary Cecal Diverticulitis Mimicking Cecal Carcinoma. J Coll Physicians Surg Pak. 2018;28(6):S75-7.

11. Tsetse C, Chaudhry SR, Jabi F, Taylor JN. Perforated cecal diverticulitis with CT diagnosis and medical management. Radiol Case Rep. 2018;14(1):30-5.

12. Socea B, Nica AA, Smaranda CA, Carâp AC, Socea LI, Dimitriu M, et al. Solitary cecum diverticulitis - A surprising diagnosis. Archives of the Balkan Medical Union. 2017;52(4):467-470

13. Fang JF, Chen RJ, Lin BC, Hsu YB, Kao JL, Chen MF. Aggressive resection is indicated for cecal diverticulitis. Am J Surg. 2003 Feb;185(2):135-40.

14. To $H$, Stella $D L$, Chandra R. Infiltrating caecal carcinoma versus appendicitis with caecal phlegmon - can computer tomography differentiate them? J Surg Case Rep. 2017;2017(2):rjx006.

15. Ferrara F, Bollo J, Vanni LV, Targarona EM. Diagnosis and Management of Right Colonic Diverticular Disease: A Review. Cir Esp. 2016;94(10):553-9.

16. Connolly D, McGookin RR, Gidwani A, Brown MG. Inflamed solitary caecal diverticulum - it is not appendicitis, what should I do? Ann R Coll Surg Engl. 2006;88(7):672-4.

17. Chiu PWY, Lam CYW, Lam SH, Wu AHW, Kwok SPY. On-Table Cecoscopy: A Novel Diagnostic Method in Acute Diverticulitis of the Right Colon. Diseases of the Colon \& Rectum. 2002;45(5):611-614.

18. Li JCM, Ng SSM, Lee JFY, Yiu RYC, Hon SSF, Leung WW, et al. Emergency laparoscopic-assisted versus open right hemicolectomy for complicated cecal diverticulitis: a comparative study. J Laparoendosc Adv Surg Tech A. 2009;19(4):479-83.

19. Graham SM, Ballantyne GH. Cecal diverticulitis. A review of the American experience. Dis Colon Rectum. 1987;30(10):821-6. 